\title{
UNREDUCED POSTERIOR DISLOCATION OF THE SHOULDER TREATED BY OPEN REDUCTION AND TRANSPOSITION OF THE SUBSCAPULARIS TENDON
}

\author{
J. K. Oyston, Royal Air Force
}

A case of posterior dislocation of the shoulder treated by open reduction and obliteration of the defect in the humeral head by implantation of the subscapularis tendon (McLaughlin 1952) is reported. The place of this operation in the treatment of posterior dislocation is discussed.

\section{CASE REPORT}

A man of thirty-two injured his left shoulder while playing rugby. The shoulder was examined and was thought to be dislocated and reduction was attempted. He was later seen at a local hospital and an antero-posterior radiograph was passed as normal. The shoulder was immobilised in a sling and he returned to the care of his own doctor. After only ten days in the sling he was allowed to move the joint. Progress was poor and he was thought to have a "frozen shoulder," which was treated by heat and active exercise.

When seen at the Royal Air Force Hospital at Cosford three months later, he complained of inability to rotate the arm outwards and of discomfort when performing other movements. On inspection the contours of the shoulders appeared symmetrical but on palpation the coracoid process was more easily felt on the affected side. The shoulder was held in medial rotation and no lateral rotation was possible (Fig. 1). Flexion and extension were possible to within 10 degrees of normal. Abduction was limited to 90 degrees, but if he was allowed to combine the movement with slight flexion he could elevate the arm to 150 degrees (Fig. 2).

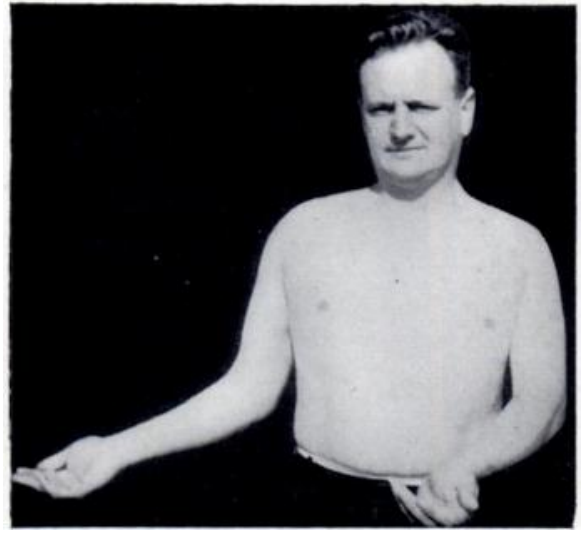

Fig. 1

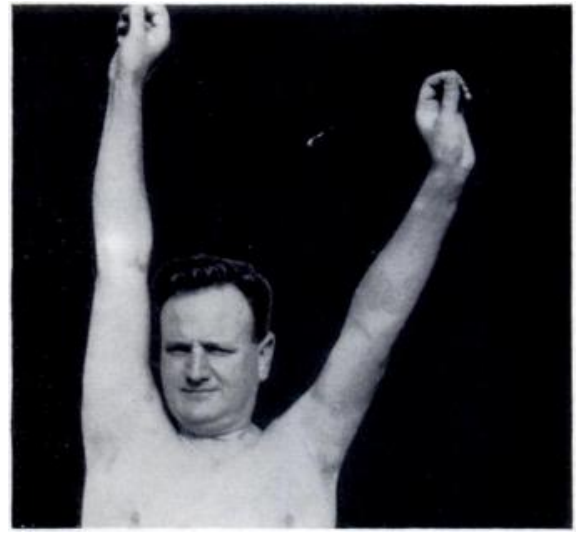

FIG. 2

Figure 1-Unreduced posterior dislocation of the left shoulder showing no lateral rotation. Figure 2-The range of abduction of the left shoulder.

An antero-posterior radiograph looked almost normal, but there was an area of decreased density in the part of the head nearest to the glenoid (Fig. 3). A radiograph taken in the vertical plane revealed the posterior dislocation and a deep V-shaped depression in the humeral head opposite the posterior glenoid rim (Fig. 4).

Operation-At operation the shoulder joint was approached through the delto-pectoral groove. The short head of the biceps and the coraco-brachialis muscles were divided close to the 
coracoid process. The subscapularis muscle was divided as close to its insertion as possible and the shoulder joint opened. The posterior glenoid rim was firmly engaged in the V-shaped defect of the humeral head, rendering reduction difficult. After reduction the humeral defect

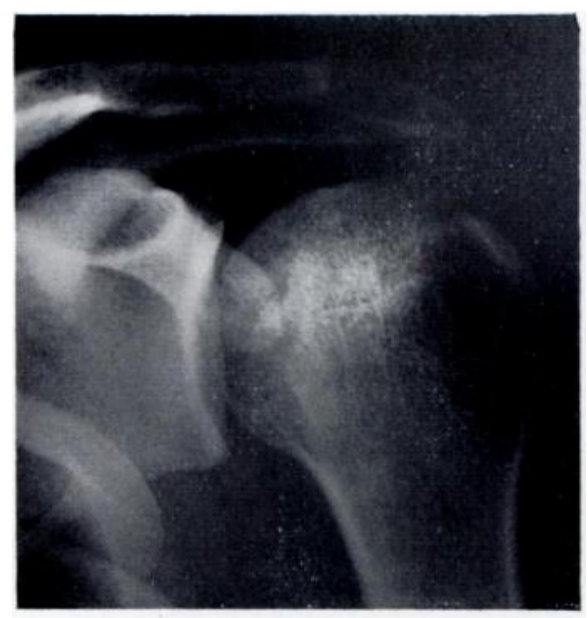

Fig. 3

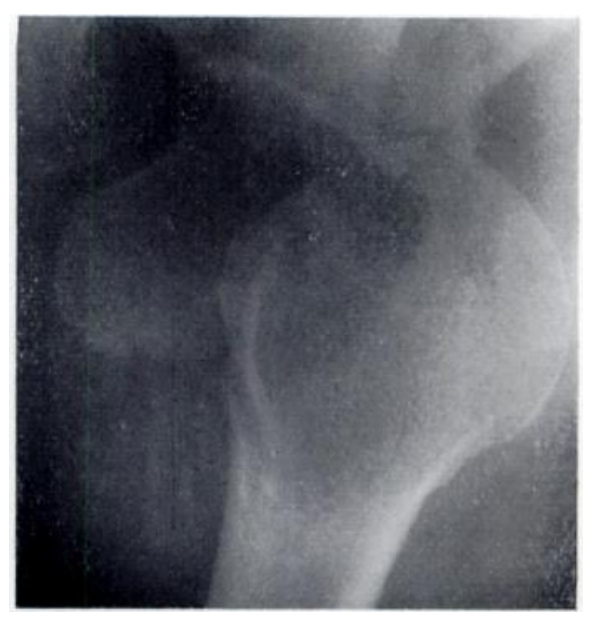

FiG. 4

Figure 3-The antero-posterior radiograph of the unreduced posterior dislocation. The dislocation could be missed, but note the area of decreased density in the part of the humeral head nearest the glenoid. Figure 4-The vertical projection radiograph showing the dislocation and the V-shaped depression in the anterior part of the humeral head.

could be clearly seen (Fig. 5). It consisted of a vertical V-shaped groove one and a quarter inches long, half an inch wide and half an inch deep situated on the anterior part of the head. The walls were smooth and covered by articular cartilage. When the arm was rotated medially redislocation occurred as the posterior rim of the glenoid slipped into the groove.

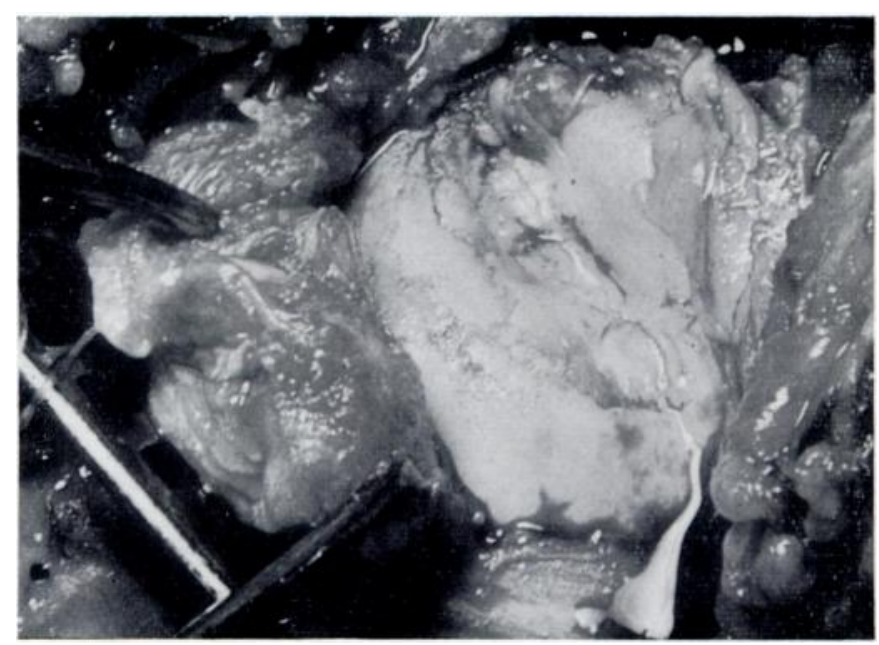

FIG. 5

The anterior part of the humeral head after reduction showing the deep groove. The subscapularis tendon grasped in the tissue forceps fits nicely into the defect.

In order to stabilise the reduction, the walls of the groove were freshened with an osteotome and the subscapularis tendon was sutured into the groove with mattress sutures passed through drill holes in the bone. The wound was closed in layers and a plaster spica applied with the 
shoulder in neutral rotation and $\mathbf{4 0}$ degrees of abduction. After five weeks the plaster was removed and active exercises were begun.

Progress-A three-quarters range of shoulder movements including medial rotation was regained within five weeks of removing the plaster. A year later he lacked only 5 degrees of abduction and 5 degrees of medial rotation. This caused him no disability either in his work as an engine fitter or in his sporting activities which included playing basketball.

\section{DISCUSSION}

Posterior dislocations of the shoulder can be classified into three groups.

Recent dislocations-Most can be reduced by manipulation under general anaesthesia. After reduction they are stable even with the humerus held in medial rotation. Immobilisation in a sling and body bandage for three weeks followed by active exercises often produces good results (McLaughlin 1952, Taylor and Wright 1952) but it is probably safer to immobilise the shoulder in a plaster spica in lateral rotation and abduction for three weeks (Watson-Jones 1956, Apley 1959). A few require open reduction.

Old untreated dislocations-The case reported is of this type. In a series of twenty-two cases reported by McLaughlin in 1952 nine presented in this way, showing how posterior dislocation can be missed by the unwary. Nevertheless, if the physical signs are looked for, the diagnosis will be made and confirmed by the vertical projection radiography technique described by Warrick in 1948.

The signs listed by previous authors include increased posterior prominence of the humeral head, decreased prominence of the head anteriorly rendering the coracoid process more easily palpable, and marked limitation of abduction and complete absence of lateral rotation.

I believe that the complete absence of lateral rotation is the most valuable sign, for in the case reported there was still complete absence of this movement despite the fact that abduction had been regained. A similar case with early recovery of abduction was reported by Nobel in 1962 .

In this group of cases there is usually considerable damage to the articular surface of the humerus. This is caused at the time of the dislocation by the posterior rim of the glenoid being thrust violently against the anterior part of the humeral head. The rim may act as a wedge and cut a V-shaped groove, or the damaged area may be more comminuted so that the whole of the anterior part of the articular surface is depressed. In either case the glenoid rim remains engaged in the humeral defect and this usually prevents closed reduction.

In the old and frail it may be best to leave the shoulder dislocated and to begin movements, but in the majority of patients open reduction is indicated. Many advise that these cases should be treated by an operation aimed at reattaching the tissues avulsed from the posterior surface of the glenoid, repairing any damage to the capsule or labrum, and reefing the capsule and the tendons of the small lateral rotators. In unstable dislocations the addition of a bone block to reinforce the posterior rim of the glenoid is advised (Watson-Jones 1956, Apley 1959, Nobel 1962).

I submit that when a clear cut V-shaped notch is present in the humeral head rendering reduction unstable in medial rotation, then obliteration of the defect and the formation of a new anterior capsule, using the subscapularis tendon, is a more logical approach to the problem. It allows treatment of the two pathological conditions: 1) the defect in the humerus is obliterated; and 2) the tissues pushed away from the posterior surface of the glenoid by the dislocated head are re-opposed to it at the time of reduction, and it is possible to freshen the surface of the bone using a small curved osteotome. Good results from this operation in a number of cases have been reported by McLaughlin (1952 and 1962). 
Recurrent dislocations-In McLaughlin's series of twenty-two posterior dislocations eight were of this type. Unless a careful history is obtained they may be mistaken for recurrent anterior dislocations. When there is doubt a radiograph taken in the vertical plane usually shows a defect on the anterior part of the humeral head confirming that the dislocation is posterior. These defects are usually shallow and the cases are best treated by posterior repair (McLaughlin 1962).

\section{SUMMARY}

1. A case of posterior dislocation of the shoulder treated by open reduction and obliteration of the defect in the head of the humerus by implantation of the subscapularis tendon is reported. 2. It is suggested that this method is indicated in cases in which there is a deep V-shaped depression on the anterior aspect of the humeral head.

I wish to thank Mr J. C. Scott for his help in preparing this paper, and the Director General of the Medical Services of the Royal Air Force for permission to publish it.

\section{REFERENCES}

Apley, A. G. (1959): A Sy'stem of Orthopaedics and Fractures, p. 283. London: Butterworth \& Co. (Publishers) Ltd.

McLaughlin, H. L. (1952): Posterior Dislocation of the Shoulder. Journal of Bone and Joint Surgery, 34-A, 584. McLaughlin, H. L. (1962): Follow-up Notes on Articles Previously Published in the Journal. Posterior Dislocation of the Shoulder. Journal of Bone and Joint Surgery, 44-A, 1,477.

Nobel, W. (1962): Posterior Traumatic Dislocation of the Shoulder. Journal of Bone and Joint Surgery, 44-A, 523.

TAYlor, R. G., and Wright, P. R. (1952): Posterior Dislocation of the Shoulder. Journal of Bone and Joint Surgery, 34-B, 624.

WARrick, C. K. (1948): Posterior Dislocation of the Shoulder Joint. Journal of Bone and Joint Surgery, 30-B, 651.

WAtSON-Jones. Sir R. (1956): Fractures and Joint Injuries. Fourth edition, p. 487. Edinburgh and London: E. \& S. Livingstone Ltd. 